Chirurg 2019 $90: 1022$

https://doi.org/10.1007/s00104-019-01040-6 Online publiziert: 23. September 2019

(c) Springer Medizin Verlag $\mathrm{GmbH}$, ein Teil von Springer Nature 2019

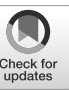

\section{Originalpublikation}

Rault-Petit B, Do Cao C, Guyetant S et al (2019) Current management and predictive factors of lymph node metastasis of appendix neuroendocrine tumors. Ann Surg 270:165-171

Hintergrund. Das operative Vorgehen bei neuroendokrinen Tumoren der Appendix (ANET) konnte auf Grundlage der Empfehlungen verschiedener internationaler Fachgesellschaften zunehmend standardisiert werden. Die Frage, welchem ANET-Patienten wegen des Risikos syn- oder metachroner Lymphknotenmetastasen (LKM) eine zweizeitig-komplettierende Rechtshemikolektomie (RHK) empfohlen werden sollte, ist jedoch weiterhin schwierig $\mathrm{zu}$ beantworten, da bislang kaum Daten eines hinreichend großen und detailgenau dokumentierten Krankengutes existieren, die eine einigermaßen verlässliche Abschätzung des Lymphknotenmetastasierungsrisikos erlauben. Die vorliegende, das umfangreiche und sorgfältig geführte Register der in ganz Frankreich rekrutierenden französischen Studiengruppe Neuroendokrine Tumoren (GTE) nutzende Untersuchung hatte daher das Ziel, prädiktive Faktoren für das Vorliegen von LKM herauszuarbeiten.

Material und Methoden. Insgesamt 403 nach 2010 in Frankreich diagnostizierte, nichtfernmetastasierte ANET wurden in die Studie eingeschlossen. Alle wesentlichen ANET-charakterisierenden morphologischen Daten der Primär-

\title{
H. Dralle
}

Sektion Endokrine Chirurgie, Klinik für Allgemein-, Viszeral- und Transplantationschirurgie, Universitätsklinikum Essen, Essen, Deutschland

\section{Risikofaktoren für Lymphknotenmetastasen bei neuroendokrinen Tumoren der Appendix}

tumoren wurden in die retrospektive Untersuchung einbezogen, ebenso die klinischen und pathologischen Befunde der durch RHK zweizeitig operierten Patienten.

Ergebnisse. 1. Primärtumoren (403): $80 \%$ der Patienten wurden wegen akuter Appendizitis operiert; $62 \%$ der ANET waren $<1 \mathrm{~cm}, 8 \%>2 \mathrm{~cm}$; $91 \%$ hatten einen Differenzierungsgrad 1 mit einem medianen $\mathrm{Ki67}$ von 1\%; 9\% waren an der Appendixbasis lokalisiert, 5\% hatten eine Mesoappendixinfiltration $>3 \mathrm{~mm}$; $15 \%$ hatten eine lymphovaskuläre, $24 \%$ eine perineurale und $8 \%$ eine $\mathrm{R} 1-\mathrm{Re}$ sektion.

2. Sekundäre RHK (80): Kein Patient verstarb. 8 Patienten (10\%) hatten Residualtumor am Zökum, 17 (21\%) waren LKM-positive; präoperativ erfolgte eine Bildgebung mit Computertomographie/ Magnetresonanztomographie bei $55 \%$ bzw. $10 \%$, ein Octreoscan bei $51 \%$ der Patienten.

3. Von insgesamt 100 Patienten mit primärer (20) oder sekundärer (80) RHK hatten 23 (6 primär, 30\%; 17 sekundär, $21 \%)$ LKM. Signifikant assoziiert mit LKM waren: Primärtumorgröße, lymphovaskuläre und perineurale Invasion. Das Vorkommen von LKM war mit einer Primärtumorgröße von $1-6,5 \mathrm{~cm}$ assoziiert, LKM-negative ANET hatten eine Größe von wenigen Millimetern bis $4 \mathrm{~cm}$.

Diskussion. Vereinfacht zusammengefasst wurde bei einem Viertel der Patien- ten eine RHK durchgeführt, ein Viertel dieser Patienten war LKM-positiv. Da die Datenquelle dieser Studie wahrscheinlich die derzeit weltweit beste darstellt, sind die Ergebnisse von großem Interesse und von Bedeutung hinsichtlich ihrer zukünftigen Berücksichtigung in den Leitlinien. Die Autoren mahnen zu recht mit Nachdruck, dass zur Entscheidungsfindung bez. einer sekundären RHK es gerade bei der kontroversen Tumorgruppe zwischen 1 und $2 \mathrm{~cm}$ von entscheidender Bedeutung ist, dass immer alle 5 hierzu wichtigen Befunde vorliegen: Lokalisation; Differenzierungsgrad; lymphovaskuläre Invasion; Mesoappendixinfiltration; R-Klassifikation. Ob die Durchführung einer RHK einen Survival-Benefit ergibt, konnte auch durch diese Studie nicht geklärt werden. Wegen der hinsichtlich des Vorkommens von LKM breiten Überlappungszone von $1-4 \mathrm{~cm}$ bei LKM-negativen (wenige Millimeter bis $4 \mathrm{~cm}$ ) bzw. LKM-positiven $(1-6,5 \mathrm{~cm})$ ANET kommt insbesondere bei Nichtdurchführung einer RHK der Bildgebung im Rahmen der Nachsorge besondere Bedeutung zu.

\section{Korrespondenzadresse}

Prof. Dr. med. Dr. h. c. mult. H. Dralle Sektion Endokrine Chirurgie, Klinik für Allgemein-, Viszeral- und Transplantationschirurgie, Universitätsklinikum Essen Hufelandstr. 55, 45147 Essen, Deutschland henning.dralle@uk-essen.de

Interessenkonflikt. H. Dralle gibt an, dass kein Interessenkonflikt besteht. 